\title{
Ovarian tumor treated by laparoendoscopic single-site surgery through the preexisting paraumbilical hernia: an initial case report
}

\author{
Naoko Sasamoto • Yoshimitsu Yamamoto $\cdot$ Kazushige Adachi
}

Received: 23 September 2012 / Accepted: 31 October 2012 / Published online: 19 December 2012

(C) Springer-Verlag Berlin Heidelberg 2012

\section{Introduction}

For benign gynecological diseases, laparoscopic surgery has become a standard method. Its advantages include fast recovery, shorter hospital stay, decreased analgesic requirements, and lower perioperative complications. In recent years, laparoendoscopic single-site (LESS) surgery has become a new branch of surgery that increases cosmetic benefits over traditional endoscopic surgery [1]. Recent reports suggest that LESS surgery has an advantage over conventional laparoscopic surgery in the aspect of postoperative pain and the use of analgesia [2-6].

Here, we present the first case of ovarian tumor treated by LESS surgery, which has been successfully applied in a patient complicated with paraumbilical hernia, using its hernia ring as a placement site for the single port.

\section{Case report}

A 59-year-old obese woman (gravida 4, para 2) was referred to the emergency department of our hospital with recurrent lower abdominal pain. Her body mass index (BMI) was $31.6 \mathrm{~kg} / \mathrm{m}^{2}$. She had a past medical history of open cholecystectomy and was treated orally for hypertension. On physical examination, there was a dimple about $3 \mathrm{~cm}$ wide just below the umbilicus. A computed tomography (CT) scan of the abdomen revealed a $9-\mathrm{cm}$ ovarian tumor, and the patient was referred to our department. The patient was diagnosed with a $7 \times 8.4-\mathrm{cm}$ right ovarian mature cystic teratoma and a paraumbilical hernia

N. Sasamoto $(\bowtie) \cdot$ Y. Yamamoto $\cdot$ K. Adachi

Department of Obstetrics and Gynecology, Minoh City Hospital,

5-7-1, Kayano,

Minoh, Osaka 562-0014, Japan

e-mail: sasamoton@gmail.com by ultrasonography, contrast CT scan, and abdominal magnetic resonance imaging (Fig. 1). The left ovary was normal in size and shape, and there was no ascites. Serum CA19-9 level was elevated at 102 units/ml (<37 units/ml), other tumor marker levels including CA-125, SCC and AFP were 14.6 units $/ \mathrm{ml}(<35$ units $/ \mathrm{ml}), 0.9 \mathrm{ng} / \mathrm{ml}$ $(<1.5 \mathrm{ng} / \mathrm{ml})$, and $4.8 \mathrm{ng} / \mathrm{ml}(<13.4 \mathrm{ng} / \mathrm{ml})$, respectively. We performed paraumbilical hernia repair and laparoscopic bilateral salpingo-oophorectomy. First, we opened the sac of the hernia and repositioned the content. A single port (SILS port; Covidien, Mansfield, MA) was then inserted into the inner ring of the paraumbilical hernia, and the abdomen was insufflated to $10 \mathrm{mmHg}$. The patient was placed in a Trendelenburg position. A rigid zerodegree laparoscope, laparoscopic forceps (SonoSurg; Olympus, Tokyo, Japan), and laparoscopic bipolar forceps (Powergrip; JSS, Osaka, Japan) were used for the laparoscopic bilateral salpingo-oophorectomy. After confirming the ureter, the infundibulopelvic ligament was coagulated by bipolar forceps and was transected. The utero-ovarian ligament was coagulated then transected in the same manner, and salpingo-oophorectomy was performed. The ovarian tumor was placed in an endoscopic pouch and removed from the pelvic cavity through the single-port site. Because the defect of the abdominal wall was only about $3 \mathrm{~cm}$ in diameter, the abdominal wall was repaired by just closing the fascia. The total operation time was $95 \mathrm{~min}$, and the amount of blood loss was very small. The right ovarian tumor contained yellow fat and pieces of hair. Pathological findings confirmed a mature cystic teratoma of the right ovary and a normal left ovary. In the postoperative period, the patient was administered antibiotic prophylaxis for 1 day as conventionally done in our hospital. She was mobilized on the day after the surgery and was discharged on the seventh postoperative day as conventionally done in our hospital. She has not experienced any complication for 1 year after the operation. 


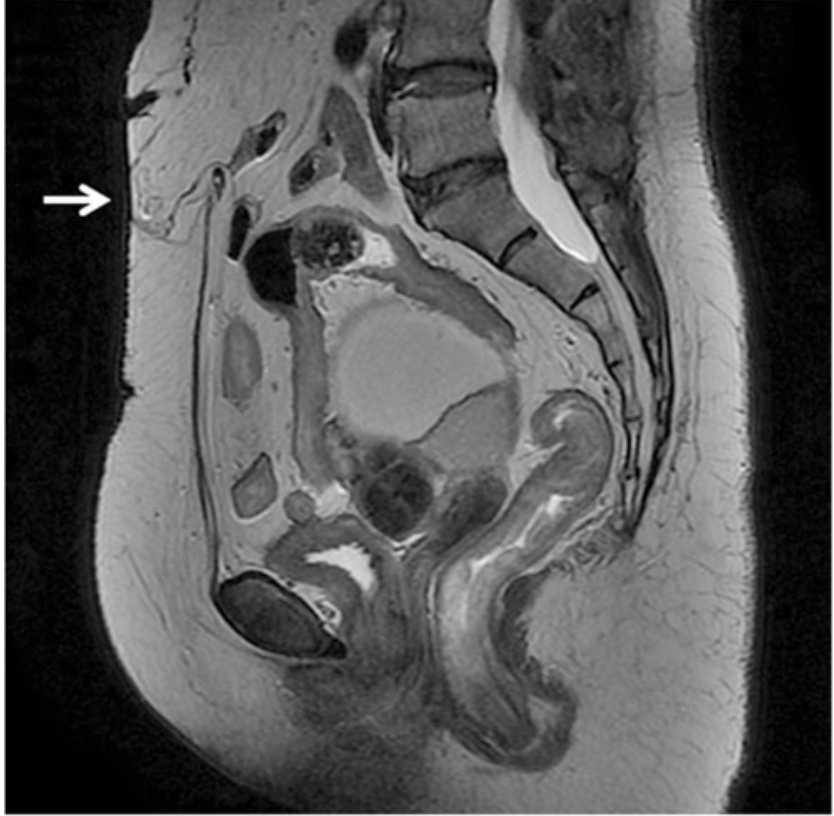

Fig. 1 A sagittal section of $T_{2}$-weighted MR image, showing the paraumbilical hernia and ovarian tumor. The arrow shows the fat of the omentum coming out from the abdominal cavity under the umbilicus

\section{Discussion}

Minimally invasive surgery has become a standard of care for the treatment of many benign gynecological diseases. From the fact that each working port carries with it an inherent risk of bleeding, infection, concordant organ damage, and hernia formation and that it decreases cosmetics, the natural goal in minimally invasive surgery is to reduce the number of ports to perform the procedure [1]. LESS surgery is an advancing technique, which involves performing laparoscopic surgery through a single, smallskin incision concealed within the umbilicus. Reports of LESS surgery from general surgery and urology have indicated that LESS surgery not only improved cosmetics, but also, in many cases, it resulted in a shorter hospitalized period and decreased postoperative analgesia requirements when compared with conventional laparoscopic approaches. Recently published data suggest feasibility, safety, and reproducibility for LESS surgery in gynecology [1]. There are reports suggesting that patients who undergo LESS surgery have less postoperative pain compared with those who received conventional laparoscopic surgery. An RCT trial was reported that LESS surgery provides an advantage over conventional laparoscopic approaches in terms of postoperative pain and need for rescue analgesia for benign adnexal diseases [2, 3].

We present the first case of the accomplishment of LESS surgery being applied to an obese patient with an ovarian tumor and paraumbilical hernia using the ring of the hernia as a placement site for the single port. To our knowledge, there is only one case report that underwent single-port cholecystectomy through the lateral ring of the left inguinal hernia [4]. There have been no reports using the current procedure for the treatment of ovarian tumor. By inserting the single port into the hernia ring, extra incision of the abdominal wall can be avoided, making the operation less invasive. Abdominal wall hernias are quite common. There are reports that the incidence of abdominal hernia is 1.5 to $2.0 \%$ [5]. Therefore, we would like to emphasize that LESS surgery is feasible, using the abdominal wall defect as a place to insert the single port.

In our case report, the patient's BMI was $31.6 \mathrm{~kg} / \mathrm{m}^{2}$. A BMI under $25 \mathrm{~kg} / \mathrm{m}^{2}$ is normal, a BMI between 25 and $29.9 \mathrm{~kg} / \mathrm{m}^{2}$ is considered overweight, and a BMI of $30 \mathrm{~kg} /$ $\mathrm{m}^{2}$ or more is defined as obesity [6]. Obesity results in a greater postoperative risk of atelectasis, pneumonia, deep venous thrombosis, pulmonary embolus, ileus, and wound infection [7]. There are reports that obese patients are associated with prolonged first flatus, later commencement of soft diet, increased administration of analgesics, and prolonged hospital stay [8]. Obesity was initially considered as a relative contraindication to operative laparoscopy. However, a growing body of evidence advocates the feasibility and safety of laparoscopic techniques rather than open surgery in obese individuals. Laparoscopy is currently considered to be a suitable technique for obese patients because it is minimally invasive, results in less postoperative pain, limits the risk of surgical site infection, leads to fewer postoperative ileus, and leads to rapid recovery and shorter period of hospitalization than open procedures [9]. Minimally invasive LESS surgery may be the best indication for obese patients if technically possible because it can further reduce the risk of postoperative wound infection by eliminating the number of trocar sites. Also, less postoperative pain may enable obese patients to resume normal activities more quickly and limit the risk of thromboembolic events and faster return of bowel functions [10]. From this point of view, we consider that LESS surgery may be a better minimally invasive approach especially to obese patients. We hope that more research data will be accumulated on this field of laparoscopy and obesity.

\section{Conclusion}

In conclusion, we demonstrate the first case of an obese patient with a paraumbilical hernia being treated to cure an ovarian tumor by laparoendoscopic single-site surgery using the ring of the hernia to place the single port. In this case report, we wish to highlight that when laparoscopic operation is considered in patients complicated with abdominal wall defect, LESS surgery using the hernia ring to place the 
single port can be considered a feasible and safe surgical approach.

Conflict of interest The authors report no conflict of interest. The authors alone are responsible for the content and writing of the paper.

\section{References}

1. Kondo W, Ribeiro R, Zomer MT (2012) Single-port laparoscopic surgery in gynecology-current status. Gynecol Surg. doi:10.1007/ s10397-012-0743-7

2. Fagotti A, Bottoni C, Vizzielli G et al (2011) Postoperative pain after conventional laparoscopy and laparoendoscopic single site surgery (LESS) for benign adnexal disease: a randomized trial. Fertil Steril 96:255-259

3. Chen YJ, Wang PH, Ocampo EJ, Twu NF, Yen MS, Chao KC (2011) Single-port compared with conventional laparoscopicassisted vaginal hysterectomy: a randomized controlled trial. Obstet Gynecol 117:906-912
4. Lukovich P, Hahn O, Tarjanyi M (2011) Single-port cholecystectomy through the lateral ring of the left inguinal hernia. Surg Innov. doi: $10.1177 / 1553350610392930$

5. Dabbas N, Adams K, Pearson K, Royle G (2010) Frequency of abdominal wall hernias: is classical teaching out of date? JRSM Short Rep 2:5

6. MacDonald KG Jr (2003) Overview of the epidemiology of obesity and the early history of procedures to remedy morbid obesity. Arch Surg 138:357-360

7. Camanni M, Bonino L, Delpiano EM, Migliaretti G, Berchialla P, Deltetto F (2010) Laparoscopy and body mass index: feasibility and outcome in obese patients treated for gynecologic diseases. J Minim Invasive Gynecol 17:576-582

8. Kim MG, Yook JH, Kim KC, Kim TH, Kim HS, Kim BS (2011) Influence of obesity on early surgical outcomes of laparoscopicassisted gastrectomy in gastric cancer. Surg Laparosc Endosc Percutan Tech 21:151-154

9. Lamvu G, Zolnoun D, Boggess J, Steege JF (2004) Obesity: physiologic changes and challenges during laparoscopy. Am J Obstet Gynecol 191:669-674

10. Chopin N, Malaret JM, Lafay-Pillet MC, Fotso A, Foulot H, Chapron C (2009) Total laparoscopic hysterectomy for benign uterine pathologies: obesity does not increase the risk of complications. Hum Reprod 24:3057-3062 